\title{
Primary care practitioner perceptions and attitudes of complementary medicine: a content analysis of free-text responses from a survey of non-metropolitan Australian general practitioners
}

\author{
Jon L. Wardle, David W. Sibbritt and Jon Adams
}

Australian Research Centre in Complementary and Integrative Medicine (ARCCIM), Faculty of Health, University of Technology Sydney, Ultimo, NSW, Australia

\begin{abstract}
Aim: This study examines GP perceptions, attitudes and knowledge of complementary medicine (CM), and to understand contextual factors that influence these perceptions, attitudes and knowledge. Background: CM use is increasing, and its influence on primary care becoming increasingly significant. Although general practitioners (GPs) often have central primary care gate-keeper roles within health systems, few studies have looked specifically at GPs' perceptions, attitudes and knowledge of CM. Methods: A questionnaire was mailed to all 1486 GPs registered as practicing in non-metropolitan areas of New South Wales. The survey included one free-text qualitative question, where respondents were invited to highlight issues associated with $\mathrm{CM}$ in their own words. Free-text responses were analyzed qualitatively using thematic analysis. Findings: In total, 585 GPs responded to the survey (adjusted response rate $40.1 \%$ ), with $152(26.0 \%)$ filling in the free-text question. Central themes which emerged were risk as a primary concern; opposition to, resistance to and the inappropriateness of complementary therapies; struggles with complexity and ambivalent tolerance. Conclusion: GPs in Australia have a wide variety of perceptions toward CM. A minority of GPs have absolute views on CM, with most GPs having numerous caveats and qualifications of individual CM. Efficacy is only one aspect of CM critically evaluated by GPs when gauging support for individual therapies - risk, alignment with medical principles and an openness to exploring new avenues of treatment where others have failed, all appear to be equally important considerations when GPs form their views around CM.
\end{abstract}

Key words: primary care; complementary medicine; interprofessional care; general practice; survey

Received 14 July 2016; revised 13 September 2017; accepted 16 September 2017;

first published online 8 February 2018

\section{Introduction}

The past few decades have seen a surge in the interest in, and utilization of complementary

Correspondence to: Dr J.L. Wardle, Australian Research Centre in Complementary and Integrative Medicine (ARCCIM), Faculty of Health, University of Technology Sydney, 235-253 Jones St, Ultimo, NSW 2007, Australia. Email: jon. wardle@uts.edu.au medicine (CM) by the public (Adams et al., 2014). $\mathrm{CM}$ includes a vast range of self-directed and practitioner-led health practices (eg, meditation, yoga, chiropractic, naturopathy) and products (eg, herbal medicine, nutritional supplements and homeopathy) that are not traditionally considered part of conventional care or the medical curriculum (Adams et al., 2014). Large-scale population-based survey findings highlight significant use of $\mathrm{CM}$ in Australia and internationally (Harris et al., 2012;

(C) Cambridge University Press 2018 
Adams et al., 2013; World Health Organization, 2013; Reid et al., 2016). In Australia the role of CM in the contemporary health system is of particular and increasing significance, with $\mathrm{CM}$ utilization being among the highest in the developed world (Bodeker and Burford, 2007; Reid et al., 2016). The estimated numbers of consultations with CM providers in Australia are similar to those of conventional providers (69.2 versus 69.3 million), with reported out-of-pocket expenditure on CM products estimated to be over $\$ 4.1$ billion annually (US\$3.8 billion) (Xue et al., 2007). The therapeutic footprint of $\mathrm{CM}$ practitioners in Australia is also substantial, with $\mathrm{CM}$ practitioners outnumbering conventional primary care providers in some areas (Wardle et al., 2011).

Although general medical practitioners (GPs) often have central primary care gate-keeper roles within health systems, relatively few studies have looked at the practice patterns, attitudes and perceptions of GPs as a specific sample group in relation to CM (Wardle et al., 2013a). High use and support of complementary medicine (CM) by GPs has been observed in Australia (Cohen et al., 2005; Pirotta et al., 2010; Janamian et al., 2011) and internationally (Amster et al., 2000; Corbin et al., 2002; Hamilton, 2003; Thomas et al., 2003; Cocconi et al., 2006; Poynton et al., 2006; Ross et al., 2006; Godin et al., 2007; Al Shaar et al., 2010). Although these studies appear to show broad support for CM by GPs in most nations - with similar factors underlying this support - it is also important to recognize significant differences across country borders. For example, homeopathy appears to be more popular and publicly accepted in European nations and New Zealand than in other countries, whereas chiropractic and naturopathy seem more popular and publicly accepted in Australia and parts of the United States than in other regions (Bodeker and Burford, 2007).

Exploring the practice patterns, attitudes and perceptions of GPs as a specific practitioner group in relation to $\mathrm{CM}$ is also particularly important, as studies of Italian and Korean physicians have indicated there do appear to be differences between groups with reference to $\mathrm{CM}$ use, attitudes and provision between primary care physicians and specialist or academic physicians (Cocconi et al., 2006; Kang et al., 2011). In a country such as Australia, where GPs as primary care physicians have an official 'gate-keeper' capacity to a variety of both medical and nonmedical services, the attitudes and perceptions of GPs are likely to have particularly significant practice and policy impact.

However, while general practice is one branch of medicine where CM has long made an impact (Adams and Tovey, 2000; Wardle et al., 2013a), this group has not gained as much research attention as specialist medical professions on this topic. Data from Australian and international studies demonstrate significant levels of interaction - via utilization and referral - between GPs and CM practitioners (Pirotta et al., 2002; Cohen et al., 2005; Poynton et al., 2006; Widmer et al., 2006; Godin et al., 2007; Stange et al., 2008; Pirotta et al., 2010; Perry et al., 2014). Given these high levels of interaction, the attitudes, perceptions and knowledge of primary care physicians toward CM deserve careful consideration. To provide additional insights into the attitudes, perceptions and use of CM by GPs practicing in rural and regional areas of Australia's largest State, the free-text open-ended questions relating to attitudes, perceptions and knowledge of CM in a survey of all GPs practicing in non-metropolitan Divisions of General Practice in New South Wales, Australia were analyzed.

\section{Methods}

A questionnaire was mailed to all 1486 GPs registered as practicing in rural and regional General Practice Divisions of NSW, with a reminder card sent after two months. The questionnaire was adapted for rural and regional use from previous Australian surveys of GP attitudes, use and practices of CM (Pirotta et al., 2002; Cohen et al., 2005). The final survey questionnaire contained 27 items and one free-text qualitative question, where respondents were invited to highlight issues associated with $\mathrm{CM}$ in their own words. Results from the quantitative parts of the survey have been published elsewhere (Wardle et al., 2013c; 2013d; 2014). This study reports findings from qualitative analysis of the free-text comments.

Results from free-text responses were transcribed and entered into an Excel spreadsheet. The text responses to the open-ended question were then analyzed qualitatively using thematic analysis, with initial codes then being used to create 
higher order categories in a cumulative manner (Liamputtong and Ezzy, 2005). Analysis was conducted by health services researchers in a public health faculty, with backgrounds in nursing, naturopathic medicine, sociology and public health. To reduce potential influence of pre-existing attitudes or experiences of $\mathrm{CM}$, the method of researcher triangulation was also employed with each member of the research team providing independent analysis of selections of the data and comparing results afterward (Patton, 2002). Quotations are used to illustrate the themes generated by this analysis, to demonstrate interpretations made. Participant labels were assigned on the order of data entry of the open-ended variable.

Ethical approval for the study was obtained from the School of Population Health Research Ethics Committee of the School of Population Health, University of Queensland and the Human Research Ethics Committee of the University of Newcastle.

\section{Results}

A total of 585 questionnaires were returned completed, with 49 questionnaires returned incomplete as 'no longer at this address;' giving an adjusted response rate of $40.7 \%$ for the entire survey. A total of $152(26.0 \%)$ respondents had filled in the free-text question. The respondent profiles of both the questionnaire as a whole and the free-text question were similar, with no significant differences in respondent demographics. Respondents had an average age between 45 and 54 years and were $53.5 \%$ male. Over threequarters of respondents $(77.8 \%, n=456)$ had completed their medical training at an Australian university. The respondent profile was broadly representative of the GP community in the study area, apart from a slight over-representation of women (Australian Medical Workforce Advisory Committee, 2005).

Many of those who responded had definite views about $\mathrm{CM}$, which were thematically coded individually with the results listed in Table 1 . The central themes which emerged were risk as a primary concern; opposition, resistance and the inappropriateness of complementary therapies; struggles with complexity and ambivalent tolerance. Sub-categories observed within these themes are also listed in Table 1.

Primary Health Care Research \& Development 2018; 19: 246-255
Table 1 Main themes and sub-categories of responses to free-text questions

\begin{tabular}{|c|c|}
\hline Themes & Category $(n)$ \\
\hline Risk as a primary concern & $\begin{array}{l}\text { Monopolization of care, use } \\
\text { as an alternative }(n=42) \\
\text { CM practitioners exploiting } \\
\text { patients ( } n=41) \\
\text { Risky for GPs to integrate } \\
\text { from medico-legal } \\
\text { perspective }(n=27)\end{array}$ \\
\hline $\begin{array}{l}\text { Opposition, resistance } \\
\text { and the inappropriateness } \\
\text { of } \mathrm{CM}\end{array}$ & $\begin{array}{l}\text { Pseudoscientific }(n=38) \\
\text { Has no evidence base }(n=37) \\
\text { CM not conducive to medical } \\
\text { practice/principles }(n=21)\end{array}$ \\
\hline Struggles with complexity & $\begin{array}{l}\text { Do not have time to learn } \\
\text { more about CM }(n=39) \\
\text { Do not know enough about } \\
\text { CM to comment or prescribe } \\
(n=39) \\
\text { CM too broad a } \\
\text { generalization }(n=34)\end{array}$ \\
\hline Ambivalent tolerance & $\begin{array}{l}\text { I have to be tolerant to retain } \\
\text { patients ( } n=38) \\
\text { Some CM are useful, other } \\
\text { are rubbish ( } n=37) \\
\text { It is hard to distinguish good } \\
\text { and bad CM ( } n=37) \\
\text { We do not know everything } \\
\text { about medicine }(n=32)\end{array}$ \\
\hline
\end{tabular}

$\mathrm{CM}=$ complementary medicine .

\section{Risk as a primary concern}

Risk was a major theme in many of the opentext responses, with participants often framing their personal attitudes and perceptions toward $\mathrm{CM}$ in relation to risk management rather than other factors such as patient preference or efficacy. Both direct (eg, drug-herb interaction, adverse events) and indirect risks (eg, delayed diagnosis) were identified by respondents, though indirect risks were generally seen as being more influential in clinical practice. CM practitioner (or product) variability or potential monopolization of care by $\mathrm{CM}$ provides was highlighted as a major issue that increased potential risks to patients. For many GPs, it was this risk, rather than risk of ineffective therapies, that was of most concern to them as clinicians, as the following quote demonstrates:

'They all [treatments] work sometimes - it's just a matter of using them judiciously. I don't think $[\mathrm{CM}]$ is the problem per se, rather 
that practitioners and patients tend to think their pet therapies will fix everything and anything'.

(GP 152)

Concerns around the risks associated with the regulatory vacuum that often surround $\mathrm{CM}$ products and services were raised by many respondents, amongst both supporters and detractors of $\mathrm{CM}$. Most respondents held that the risks of $\mathrm{CM}$ were maximized due to the variability and heterogeneity in standards, practices and treatments, which in their opinion was related in a large part to this regulatory vacuum.

The risks from integrating or utilizing $\mathrm{CM}$ were not seen to be limited to patients, but also to the GPs themselves. For some GPs, resistance to further integration of $\mathrm{CM}$ was not necessarily due to their own perceptions on whether integration was appropriate based on patient risk, but rather perceived as a product of external factors, particularly legislative and regulatory concerns that were risky to GPs themselves. As the following response indicates, this could lead to GPs resisting referral, even in instances where they would otherwise be supportive:

'Fear of litigation over risks associated with cervical manipulation stop me referring to chiropractors or osteopaths on paper. I do believe they have great success with certain conditions'.

(GP 80)

\section{Opposition, resistance and the inappropriateness of complementary therapies}

Some respondents relayed their concerns about $\mathrm{CM}$ purely in evidence-based terms, stating that the only issue they had with most CM was that it did not abide by the principles of evidence-based medicine, and as such, was by default not appropriate for practice or integration into medical practice. The following quote illustrates the attitude presented by many respondents:

'There is only evidence-based medicine and non-evidence-based medicine. If you can show me the evidence for it I would even start using crystal therapy tomorrow'.

(GP 110)

For others, the opposition to $\mathrm{CM}$ ran deeper, indicating that even if some $\mathrm{CM}$ disciplines were to adopt evidence-based principles of practice, the historical roots of such $\mathrm{CM}$ in pseudoscience and unorthodox philosophy should disqualify them from further integration, as demonstrated by the following illustrative quote:

'Homoeopathy is bunk. Acupuncture is grossly overrated, and is not popular in China. TCM [Traditional Chinese Medicine] is only used by the poor in China. Nutriceuticals [sic] is a scam. Chiropractic is trying to position itself as more scientific but historically it's based on pseudoscience'.

(GP 78)

Respondent opposition to $\mathrm{CM}$ sometimes resulted in refusal to discuss $\mathrm{CM}$ with patients. Some respondents suggested that discussion of $\mathrm{CM}$ in a conventional medical consultation may give $\mathrm{CM}$ an undeserved imprimatur. For other GPs, their opposition to CM simply meant that CM was a topic to be 'kept off the table,' as illustrated by the following quote:

'I find if I don't ask patients about their [CM] they won't tell me about it. This suits me fine. I don't want to know what they're using as it's all garbage, and they know I'll just tell them to stop it anyway'.

(GP 11)

For some GPs, opposition to CM was absolute and was not solely directed at those that were trained in $\mathrm{CM}$ disciplines. Even conventionally trained practitioners drew the ire of some of their colleagues if they dabbled in CM provision, as the following quote demonstrates:

'Unfortunately CM practitioners aren't the only charlatans out here. I know of many doctors who practice questionable therapies and I'm never ceased to be amazed by the rubbish that pharmacists have the gall to put on their shelves. It's the opium for the masses'.

(GP 117)

Some stated opposition appeared to be associated not with open resistance to $\mathrm{CM}$, but frustrations among GPs that many $\mathrm{CM}$ practitioners were free of the accountability and scrutiny that occurred in general practice. It was felt by many participants that $\mathrm{CM}$ therapists practiced in a regulatory vacuum (and were therefore able to 
practice without restrictions on autonomy), and had a patient-base that was more forgiving and less critical of their practice than was observed in general practice, as evidenced by the following quote:

'Locally, patients expect orthodox medicine for free but pay for quackery and unproven therapy. They don't question ridiculous claims that are unproven. Yet question much of what I and other orthodox medicine offer'.

(GP 82)

\section{Struggles with complexity}

For many GPs, CM use among patients was acknowledged as being a practical modern reality, irrespective of whether they personally perceived merit in the individual $\mathrm{CM}$ practiced. $\mathrm{CM}$ was perceived as being both too prevalent to ignore, but also too large a field to fully embrace or evaluate. As such, CM use was considered to be a matter for patients and CM practitioners, rather than the respondents themselves:

'I assume all my patients are using [CM] and pragmatically don't mind as if they get benefit that's great. However, I'm not interested in learning more as I already have enough trouble keeping up to do date with orthodox medicine'.

(GP 22)

For some GPs, the diversity of CM was said to be overwhelming, and in some instances may result in them not using $\mathrm{CM}$, or feeling uneasy about using CM, even in clinical scenarios where they thought it appropriate, as can be observed in the following quote:

'I know too little to be confident about prescribing or recommending something'.

(GP 29)

Part of this complexity was the fact that there was little distinction among respondents of medicines, therapies and practitioners when constructing personal definitions of CM. However, many GPs did note the fraught nature of referring to $\mathrm{CM}$ as a homogenous entity:

'Some $[\mathrm{CM}]$ are good, some are not. There's too much variability to term everything under the one moniker'.

(GP 34)
Some respondents highlighted that the complexities of CM not only affected their perception of individual CM therapies, but also changed their support for a specific CM in differing contexts, where even $\mathrm{CM}$ that was generally poorly perceived by GPs could be deemed appropriate in specific circumstances:

'My opinions on what is effective in CM are hard to generalize into categories. I find some herbal remedies effective, many are rubbish. Likewise I disagree with the general principles of homeopathy but have recommended rescue remedy before. I believe increased communication between practitioners of CM and GPs should be encouraged. It seems slow-going'.

(GP 69)

Other GPs were concerned about the practical implications of such broad CM categorization. Many explained the inclusion of well-accepted or perceivably feasible $\mathrm{CM}$ with fringe modalities as potentially dangerous, and as a way of giving some $\mathrm{CM}$ undeserved legitimacy. This interpretation is demonstrated in the GP quote below:

"Involving all "alternative medicine" under one umbrella such as [CM] is an attempt to give some sham modalities such as homeopathy credibility by attempting comparison to massage/meditation etc'.

(GP 83)

\section{Ambivalent tolerance}

Few GPs expressed complete support for inclusion, or further integration of CM. Many GPs did offer supportive statements, though these were often couched with caveats or caution. As such, many respondents exhibited a form of ambivalent sometimes belligerent - tolerance to $\mathrm{CM}$ use, whereby the validity of CM use was still questioned, but respondents adopted a more pragmatic view of $\mathrm{CM}$, one which was neither wholly positive nor negative. Pragmatism (influenced by patient beliefs), rather than explicit support for $\mathrm{CM}$ often engendered a tolerant or open attitude toward CM for many GPs, as the following quote illustrates:

'My community is very alternative. It is important to be open and not judgmental to ensure compliance and openness in patients. 
They will choose the $[\mathrm{CM}]$ practitioner over me if there was any hint of judgment or hostility'.

(GP 43)

In some instances some GPs rejected the notion that some aspects of CM were in fact 'alternative' or 'complementary' at all, but a part of medicine and wanted these differentiated from other CM modalities and practices that they deemed more fringe or unorthodox. Nutritional medicine was often an example given, as indicated in the quote below:

'Nutritional medicine is part of proper medicine, not complementary. Some [CM] therapists go "too far", even if they do have useful skills. There needs to be adequate regulation as there is a lot of difference between practitioners and groups'.

(GP 35)

Other practitioners relayed the potential for CM to be used as placebo in practuce, particularly in the case of 'heart-sink' patients who were unable to be helped by more conventional treatments. As the following quote demonstrates, in this sense they often supported CM use in specific circumstances, even when they had less positive views about CM more generally:

' $[\mathrm{CM}]$ can be useful to generate a placebo response that the GP cannot ethically prescribe - e.g. often useful for anxiety/depression of mild variety. But some of the guff that gets referred back "the naturopath wants you to test/treat my yeast infection" etc. are both aggravating and entertaining'.

(GP 6)

\section{Discussion}

This study provides interesting contextualization of previous descriptive survey findings around medical practitioner utilization and integration of $\mathrm{CM}$ practices and offers new insights to complement other work regarding GPs' perceptions of $\mathrm{CM}$ and the interface with primary health care. Such work has focused largely on quantitative reporting of figures support for, referral to and practice of individual CMs (Wardle et al., 2013b; 2013c; 2013d; 2014). Findings from this study suggest that there are few default positions of GPs relating to CM. GP perceptions toward CM appear to be accompanied by a number of caveats and qualifying statements, and there is significant diversity of view among GPs on most issues relating to $\mathrm{CM}$. The only group of GPs in this study which appeared to hold definitive views on $\mathrm{CM}$ was the minority of GPs who ideologically opposed CM on all levels, who suggested CM was pseudoscientific and incompatible with medical practice. GPs who supported CM, or GPs who stated they could support it in specific contexts, usually only did so conditionally (eg, using it as a benign placebo, rather than being convinced of its effectiveness). For most respondents their attitudes and perceptions of $\mathrm{CM}$ in specific circumstances was framed in a discourse of risk, with risk minimization being seen as more important than maximizing benefits of $\mathrm{CM}$ use, possibly due to an overarching skepticism of CM efficacy. Efficacy seems to be only one aspect of CM critically evaluated by GPs when gauging their support for individual $\mathrm{CM}$ therapies - risk, alignment with medical principles and openness to exploring new avenues of therapies where others have failed, all appear to be equally important considerations. However, most contemporary commentary around the issues surrounding $\mathrm{CM}$ and primary practice appears to focus on whether CM is effective enough to integrate into medical practice (Ernst, 2016). Findings from this study suggest that more nuanced discussion relating to the policy and practice interface between $\mathrm{CM}$ and primary care is necessary to ensure it adequately reflects the issues experienced at the coalface of primary care practice.

The findings from this study seem broadly consistent with other studies of Australian GPs, which show generally high levels of support for CM, yet also show a significant minority of GPs harboring negative attitudes or heightened skepticism toward CM (Easthope et al., 2000; Hall and GilesCorti, 2000; Cohen et al., 2005). The prominence of risk as a factor in attitudes and perceptions of $\mathrm{CM}$ in practice in our study is also supported by analysis of Australian biomedical journal content on CM, which showed that most CM content published in those journals was framed under a discourse of risk (Lewis, 2013). Our work adds clarity and context to these previous studies, by offering insights into the factors that underpin varying 
levels of support for CM. The qualifications and caveats around issues such as efficacy, for example, the support for CM use by skeptical GPs in 'heartsink' patients where other options have failed, or that efficacy may not be the primary concern in relation to $\mathrm{CM}$, also mirrors earlier international qualitative work of $\mathrm{CM}$ in general practice, which uncovers a similarly nuanced approach to CM integration (Adams, 2003; Brien et al., 2008; Jarvis et al., 2015). Just as there may be no such thing as a 'typical' GP, these findings would also suggest that there appears to be no such thing a 'typical' GP view of $\mathrm{CM}$. These findings, and the reasons that underlie such differing views, suggest that information tools and strategies to encourage GP discussion around $\mathrm{CM}$ may need to be tailored to a wide variety of views, rather than assuming a 'one-size fits all' strategy. This would necessitate a variety of communication and dissemination strategies for GP CM education, rather than the development of resources aimed at a single GP market. Such development should also account for the significant heterogeneity of CM. Although often defined as a discrete and homogenous group, there is significant heterogeneity in CM approaches, which according to participants in our study included therapies identified as 'fringe' or 'pseudoscientific' as well as therapies and practices that are considered relatively mainstream in Australia (eg, chiropractic is covered by the Australian public health insurer Medicare upon referral by a GP). There may also be significant regional differences in CM utilization and prevalence that influence GP perceptions. However, relatively few respondents in our study acknowledged this heterogeneity, often discussing $\mathrm{CM}$ as a homogenous entity.

Non-disclosure of CM use in general practice was identified as a significant issue in our study, which may pose potential direct and indirect risks to patient care (Wardle and Adams, 2014). An international review of $\mathrm{CM}$ disclosure to conventional medical practitioners found that, although risks of non-disclosure are known to be significant, patients are generally hesitant to disclose CM use to medical practitioners, even when prompted, and medical practitioners routinely do not initiate discussion around CM use in their patients (Robinson and McGrail, 2004). Our study suggests simply highlighting well-known risks of non-disclosure to those GPs opposing CM appears not to encourage discussion of CM use with patients, even when those GPs may acknowledge the importance of understanding $\mathrm{CM}$ use to ensure optimal patient care, and as such offers insights into why addressing CM non-disclosure between GPs and patients remains difficult. Our study suggests $\mathrm{CM}$ use by patients may be perceived by some GPs to be an issue best ignored due to it being 'too complex' or not being as important as other general practice issues. This suggests that not only does CM information for GPs need to be tailored for a wide variety of views, but it also needs to be presented in an accessible and clinician-friendly manner. Development of tailored and flexible CM information and clinical tools may assist GPs to better navigate the interface between $\mathrm{CM}$ and general practice, but current tools do not appear to be fulfilling this role. Additionally, highlighting the ways in which encouraging $\mathrm{CM}$ discussion can be important in improving patient care beyond reducing direct risks (eg, using $\mathrm{CM}$ discussion to establish trust), may also help to reduce the rate of non-disclosure of $\mathrm{CM}$ use in general practice.

The large and varied study area was chosen to be broadly representative of Australian general practice demographics. Nevertheless, the location of the GPs in this study should be considered when generalizing the study's results to the broader Australian or international general practice population, or to other medical specialties. The nonmetropolitan nature of respondents may impact the findings of this study, given that CM use is observed to be highest in rural and regional areas in Australia (Wardle et al., 2012). Further research in other settings is warranted, particularly given the continued significance of $\mathrm{CM}$ in primary care practice, and the relative paucity of recent studies in this area.

Other limitations of the study include selfselection which may have resulted in a response bias, due to $\mathrm{CM}$ being a controversial issue in general practice, and those with particular strong views may have been more likely to participate. This effect may be particularly pronounced in the free-text response section which required additional respondent effort beyond other questions in the survey (Rich et al., 2013). The response rate is typical for large-scale GP surveys on $\mathrm{CM}$ conducted in Australia over the past decade, which have reported response rates of between 29 and 58\% (Cohen et al., 2005; Pirotta et al., 2010; 
Janamian et al., 2011), and compares well with general surveys of Australian GPs, which routinely have difficulty receiving response rates of over $30 \%$ (Bonevski et al., 2011). While the free-text nature of this survey cannot replicate depth of more-focused qualitative exploration, the broad range of perspectives gathered from this open-ended question nested within a larger quantitative survey represent an important series of insights which may otherwise have not been exposed.

\section{Conclusion}

GPs in Australia have a wide variety of perceptions toward CM. Only a subset of those GPs who oppose CM have absolute views toward the topic, with most GPs having numerous caveats and qualifications of individual CM. Efficacy seems to be only one aspect of CM critically evaluated by GPs when they gauge their support for individual therapies - risk, alignment with medical principles, and an openness to exploring new avenues of treatment where others have failed, all appear to be equally important considerations when GPs form their views around CM.

\section{Acknowledgments}

The authors would like to acknowledge the Divisions of General Practice for their support in recruitment for this study.

\section{Financial Support}

This work was funded by the National Health and Medical Research Council and the University of Queensland Research Excellence Foundation.

\section{Ethical Standards}

Ethical approval for the study was obtained from the School of Population Health Research Ethics Committee of the School of Population Health, University of Queensland (JW130508) and the Human Research Ethics Committee of the University of Newcastle (H0080344).

\section{Conflicts of Interest}

None.

\section{References}

Adams, J. 2003: The positive gains of integration: a qualitative study of GPs' perceptions of their complementary practice. Primary Health Care Research and Development 4, 155-62.

Adams, J., Andrews, G., Barnes, J., Magin, P. and Broom, A. (editors) 2014: Traditional, complementary and integrative medicine: an international reader. Basingstoke: Palgrave McMillan.

Adams, J., Sibbritt, D., Broom, A., Loxton, D., Wardle, J., Pirotta, M. and Lui, C.W. 2013: Complementary and alternative medicine consultations in urban and nonurban areas: a National Survey of 1427 Australian women. Journal of Manipulative and Physiological Therapeutics 36, 12-19.

Adams, J. and Tovey, P. 2000: Complementary medicine and primary care: towards a grass-roots focus. In Tovey, P., editor, Contemporary primary care: the challenges of change. Milton Keynes: Open University Press, 167-83.

Al Shaar, I., Ismail, M., Yousuf, W. and Salama, R. 2010: Knowledge, attitudes and practice of general practitioners towards complementary and alternative medicine in Doha, Qatar. Eastern Mediterranean Health Journal 16, 522-27.

Amster, M., Cogert, G., Lie, D. and Scherger, J. 2000: Attitudes and use of complementary and alternative medicine by California family physicians. International Journal on Grey Literature 1, 77-81.

Australian Medical Workforce Advisory Committee. 2005: The general practice workforce in Australia: supply and requirements to 2013, AMWAC Report 2005.2, Sydney.

Bodeker, G. and Burford, G. (editors) 2007: Traditional, complementary and alternative medicine: policy and public health perspectives. London: Imperial College Press.

Bonevski, B., Magin, P., Horton, G., Foster, M. and Girgis, A. 2011: Response rates in GP surveys: trialling two recruitment strategies. Australian Family Physician 40, 427-30.

Brien, S., Howells, E., Leydon, G.M. and Lewith, G. 2008: Why GPs refer patients to complementary medicine via the NHS: a qualitative exploration. Primary Health Care Research \& Development 9, 205-15.

Cocconi, G., Caminiti, C., Capriglia, S., Gennari, M., Minari, R., Schianchi, P. and D'aloia, T. 2006: Attitudes to, and practice of, unconventional medicine by physicians in Italy. European Journal of Internal Medicine 17, 32-37.

Cohen, M., Penman, S., Pirotta, M. and Da Costa, C. 2005: The integration of complementary therapies in Australian general practice: results of a national survey. Journal of Alternative \& Complementary Medicine 11, 995-1004.

Corbin Winslow, L. and Shapiro, H. 2002: Physicians want education about complementary and alternative medicine to enhance communication with their patients. Archives of Internal Medicine 162, 1176-81.

Easthope, G., Tranter, B. and Gill, G. 2000: General practitioners' attitudes toward complementary therapies. Social Science \& Medicine 51, 1555-61. 
Ernst, E. 2016: Integrative medicine: more than the promotion of unproven treatments? Medical Journal of Australia 204, 174.

Godin, G., Beaulieu, D., Touchette, J., Lambert, L. and Dodin, S. 2007: Intention to encourage complementary and alternative medicine among general practitioners and medical students. Behavioral Medicine 33, 67-77.

Hall, K. and Giles-Corti, B. 2000: Complementary therapies and the general practitioner. A survey of Perth GPs. Australian Family Physician 29, 602-6.

Hamilton, E. 2003: Exploring general practitioners' attitudes to homoeopathy in Dumfries and Galloway. Homoeopathy 92, 190-94.

Harris, P.E., Cooper, K.L., Relton, C. and Thomas, K.J. 2012: Prevalence of complementary and alternative medicine (CAM) use by the general population: a systematic review and update. International Journal of Clinical Practice 66, 924-39.

Janamian, T., O'rourke, P., Myers, S. and Eastwood, H. 2011: Information resource needs and preference of Queensland general practitioners on complementary medicines: result of a needs assessment. Evidence Based Complementary and Alternative Medicine 2011, 810908, https://doi.org/10.1155/ 2011/810908.

Jarvis, A., Perry, R., Smith, D., Terry, R. and Peters, S. 2015: General practitioners' beliefs about the clinical utility of complementary and alternative medicine. Primary Health Care Research \& Development 16, 246-53.

Kang, S., Ha, T., Cho, B., Lee, S., Han, D., Lee, K. and Hahn, S. 2011: Comparison of knowledge, attitude, and experience about complementary and alternative medicine between primary care physicians and academic physicians in Korea. Journal of the Korean Medical Association 54, 217-29.

Lewis, M. 2013: Risk and efficacy in biomedical media representations of herbal medicine and complementary and alternative medicine (CAM). Journal of EvidenceBased Complementary and Alternative Medicine 16, 210-17.

Liamputtong, P. and Ezzy, D. 2005. Qualitative research methods. Melbourne: Oxford University Press.

Patton, M. 2002. Qualitative research \& evaluation methods. Thousand Oaks, CA: Sage.

Perry, R., Dowrick, C. and Ernst, E. 2014: Complementary medicine and general practice in an urban setting: a decade on. Primary Health Care Research \& Development 15, 262-67.

Pirotta, M., Farish, S., Kotsirilos, V. and Cohen, M. 2002: Characteristics of Victorian general practitioners who practise complementary therapies. Australian Family Physician 31, 1133-38.

Pirotta, M., Kotsirilos, V., Brown, J., Adams, J., Morgan, T. and Williamson, M. 2010: Complementary medicine in general practice - a national survey of GP attitudes and knowledge. Australian Family Physician 39, 946-50.

Poynton, L., Dowell, A., Dew, K. and Egan, T. 2006: General practitioners' attitudes towards (and use of) complementary and alternative medicine: a New Zealand nationwide surrey. New Zealand Medical Journal 119, U2361.

Reid, R., Steel, A., Wardle, J., Trubody, A. and Adams, J. 2016: Complementary medicine use by the Australian population: a critical mixed studies systematic review of utilisation, perceptions and factors associated with use. BMC Complementary and Alternative Medicine 16, 176.

Rich, J., Chojenta, C. and Loxton, D. 2013: Quality, rigour and usefulness of free-text comments collected by a large population based longitudinal study - ALSWH. PLOS ONE 8, e68832.

Robinson, A. and McGrail, M. 2004: Disclosure of CAM use to medical practitioners: a review of qualitative and quantitative studies. Complementary Therapies in Medicine 12, 90-98.

Ross, S., Simpson, C. and McLay, J. 2006: Homoeopathic and herbal prescribing in general practice in Scotland. British Journal of Clinical Pharmacology 62, 647-52.

Stange, R., Amhof, R. and Moebus, S. 2008: Complementary and alternative medicine: attitudes and patterns of use by German physicians in a national survey. Journal of Alternative and Complementary Medicine 14, 1255-61.

Thomas, K., Coleman, P. and Nicholl, J. 2003: Trends in access to complementary or alternative medicines via primary care in England: 1995-2001 results from a follow-up national survey. Family Practice 20, 575-77.

Wardle, J. and Adams, J. 2014: Indirect and non-health risks associated with complementary and alternative medicine use: an integrative review. European Journal of Integrative Medicine 6, 409-22.

Wardle, J., Adams, J., Broom, A. and Sibbritt, D. 2013a: Examining the relationship between complementary and integrative medicine and rural general practice: a focus upon health services research. In Adams, J., Magin, P. and Broom, A., editors, Primary care and complementary and integrative medicine: practice and research. London: Imperial College Press, 115-32.

Wardle, J., Adams, J., Magalhaes, R.J. and Sibbritt, D. 2011: Distribution of complementary and alternative medicine (CAM) providers in rural New South Wales, Australia: a step towards explaining high CAM use in rural health? Australian Journal of Rural Health 19, 197-204.

Wardle, J., Adams, J. and Sibbritt, D. 2013b: Homeopathy in rural Australian primary health care: a survey of general practitioner referral and practice in rural and regional New South Wales, Australia. Homeopathy 102, 199-206.

Wardle, J., Lui, C.-W. and Adams, J. 2012: Complementary and Alternative Medicine in rural communities: current research and future directions. Journal of Rural Health 28, 101-12.

Wardle, J., Sibbritt, D. and Adams, J. 2013c: Referral to Chinese medicine practitioners in Australian primary care: a survey of New South Wales rural and regional general practitioners. Chinese Medicine 8.

Wardle, J., Sibbritt, D. and Adams, J. 2013d: Referrals to chiropractors and osteopaths: a survey of general 
practitioners in rural and regional New South Wales, Australia. Chiropractic and Manual Therapies 21, 5.

Wardle, J.L., Sibbritt, D.W. and Adams, J. 2014: The interface with naturopathy in rural primary health care: a survey of referral practices of general practitioners in rural and regional New South Wales, Australia. BMC Complementary and Alternative Medicine 14, 238.

Widmer, M., Herren, S., Donges, A., Marian, F. and Busato, A. 2006: Complementary and conventional medicine in
Switzerland: comparing characteristics of general practitioners. Forschende Komplementarmedizin 13, 234-40.

World Health Organization. 2013: WHO traditional medicine strategy 2014-2023. Geneva: World Health Organization.

Xue, C., Zhang, A., Lin, V., Da Costa, C. and Story, D. 2007: Complementary and alternative medicine use in Australia: a national population-based survey. Journal of Alternative and Complementary Medicine 13, 643-50. 